\title{
Neoteric advancement in TB drugs and an overview on the anti-tubercular role of peptides through computational approaches
}

\author{
Ameer Khusro $^{\mathrm{a}}$, Chirom Aarti ${ }^{\mathrm{a}}$, Alberto Barbabosa-Pliego ${ }^{\mathrm{b}}$, Abdelfattah Z.M. Salem ${ }^{\mathrm{b}}$, \\ ${ }^{\text {a }}$ Research Department of Plant Biology and Biotechnology, Loyola College, Nungambakkam, Chennai 600034, Tamil Nadu, India \\ ${ }^{\mathrm{b}}$ Facultad de Medicina Veterinaria y Zootecnia, Universidad Autónoma del Estado de Mexico, Toluca, México
}

\section{A R T I C L E I N F O}

\section{Keywords:}

Anti-tubercular drugs

Anti-tubercular peptides

Computational tools

Mycobacterium tuberculosis

Tuberculosis

\begin{abstract}
A B S T R A C T
Tuberculosis (TB) is a devastating threat to human health whose treatment without the emergence of drug resistant Mycobacterium tuberculosis (M. tuberculosis) is the million-dollar question at present. The pathogenesis of $M$. tuberculosis has been extensively studied which represents unique defence strategies by infecting macrophages. Several anti-tubercular drugs with varied mode of action and administration from diversified sources have been used for the treatment of TB that later contributed to the emergence of multidrug-resistant tuberculosis (MDR-TB) and extensively drug-resistant tuberculosis (XDR-TB). However, few of potent anti-tubercular drugs are scheduled for clinical trials status in 2017-2018. Peptides of varied origins such as human immune cells and non-immune cells, bacteria, fungi, and venoms have been widely investigated as anti-tubercular agents for the replacement of existing anti-tubercular drugs in future. In the present review, we spotlighted not only on the mechanisms of action and mode of administration of currently available anti-tubercular drugs but also the recent comprehensive report of World Health Organization (WHO) on TB epidemic, diagnosis, prevention, and treatment. The major excerpt of the study also inspects the direct contribution of different computational tools during drug designing strategies against $M$. tuberculosis in order to grasp the interplay between anti-tubercular peptides and targeted bacterial protein. The potentiality of some of these anti-tubercular peptides as therapeutic agents unlocks a new portal for achieving the goal of end TB strategy.
\end{abstract}

\section{Introduction}

Tuberculosis (TB) is one of the oldest and deadliest airborne asymptomatic tropical diseases in the world, caused by $M$. tuberculosis (Mtb), infecting one third of the global populace. The disease remains a devastating health issue in developing countries. TB can infect any parts of the body but generally affects the lungs. Development of TB outside the lungs is termed as extra-pulmonary TB and occurs mainly in young adults and immune suppressed persons. The "latent tuberculosis" is a special type of TB when the infections are devoid of any symptoms and progress to active disease if left untreated. In fact, in latent TB, Mtb remains inside the body in inactive state and becomes active later on. This type of TB is not contagious and persons affected with latent TB do not spread the disease and diagnosed by tuberculin skin test (TST) or blood tests. The active TB can be transmitted to other person and diagnosis of active TB is solely relied on chest X-rays, microscopic observation, and culturing the body fluids. The chronic cough, chillness, blood-containing sputum, fever, night sweats, chest pain, fatigue, nails clubbing, and weight loss are the classic symptoms of active TB.
"Osseous tuberculosis" is another type of tuberculosis where the infection spreads to the bones. The bursting of a tubercular abscess through skin causes tuberculous ulcer.

At present, the emergence of multidrug-resistant tuberculosis (MDRTB) and extensively drug-resistant tuberculosis (XDR-TB) has attracted attention to attain effective prevention, durable cure, and treatment of the distending problems of TB. The major obstacle in the treatment of TB is the unique mode of pathogenicity of Mtb. In fact, Mtb infects human pulmonary macrophage and escapes from the phagocytosis process. Mtb utilizes macrophages for its own replication process and survives under the immoderate radical environment. In a nutshell, Mtb arrests the maturation mechanism of phagosomes and prevents the phagosomal acidification. Further, it inhibits not only the synthesis of lysosomes and phagosomes complex but also impairs the apoptosis of macrophage as well as represses the antimicrobial actions, thereby causing bacterial escape from the phagosomes. It is totally clear that Mtb has potentiality to survive in the unfavourable conditions created by host macrophage during the infection.

In addition to this, the association of TB with HIV infection is

\footnotetext{
* Corresponding author.

E-mail address: asalem70@yahoo.com (A.Z.M. Salem).
} 
another considerable issue that caused 0.32 million mortality worldwide [1]. These epidemiological problems shed light on the relevance of the immune system to control TB and create a public health problem among large population of human.

From the last century, BCG vaccine has shown some protective efficacy in combating serious TB [2]. But the lack of protecting pulmonary infections is the poor aftermath of this therapy and has been called into the million-dollar question. In this regard, several anti-tubercular drugs of distinct origins have been developed and used from last few decades.

\section{Anti-tubercular drugs: An overview}

The treatment of TB consists of a multi-drug regimen with a long duration of 6-8 months. The preferred first-line anti-tubercular drugs are isoniazid, rifampicin, pyrazinamide, and ethambutol. Second-line drugs viz. streptomycin, kanamycin, fluoroquinolones, ethionamide, and p-aminosalicylic acid are used when the bacterial strain becomes resistant to one or more of first-line drugs. In general, second-line drugs are less effective and more toxic compared to the first-line drugs. Surprisingly, TB therapy is quite different from the treatment of other diseases due to the unique properties of MTb. Firstly, the treatment involves a combination of several antibiotics to prevent the selection of a resistant strain. Secondly, the antibiotics must be provided for a minimum period of 6 months in order to eradicate the pathogen so that there is no possibility of relapse after the completed course of treatment. Finally, but most importantly, patients should be regularly monitored throughout the treatment courses in order to observe any drug-associated induced major/minor toxicities.

The pathogenesis of Mtb is inhibited by available anti-tubercular drugs through various modes of action as shown in Fig. 1. In fact, TB is curable, provided that the drug regime is followed diligently and thus, avoiding the emergence of MDR-TB. Currently, there are several drugs available for the treatment of TB. Table 1 shows not only the list of drugs that are commonly used to cure TB but also their mode of action.

\section{New anti-tubercular drugs: bedaquiline and delamanid in brief}

From the last five decades, only two new anti-tubercular drugs were approved and released. Bedaquiline is a first-in-class diarylquinoline compound that showed therapeutic potency against drug-sensitive and drug resistant Mtb with a novel mode of action including the inhibition of bacterial ATP synthase (by binding to subunit $\mathrm{c}$ of the enzyme), the enzyme used by bacteria to generate energy. Hards et al. [25] clearly showed the uncoupling process induced by bedaquiline. In a typical Mtb cell, the majority of ATP synthesis is respiratory, driven by the proton-motive force (PMF). The binding of bedaquiline to the c-ring most likely perturbs the a-c subunit interface, thereby causing an uncontrolled proton leak uncoupled from ATP synthesis and resulting in a futile proton cycle (Fig. 2). This drug is also known by the trade name of 'Sirturo'. Bedaquiline is used in combination with other TB drugs to treat pulmonary TB in adults with MDR-TB. It should only be used when an effective TB treatment cannot otherwise be provided and other drugs are showing severe toxicity to patients. The use of bedaquiline is avoided for the treatment of extra-pulmonary TB, drug sensitive TB, latent TB infections, and infection caused by mycobacteria other than Mtb. The recommended dose is $400 \mathrm{mg}$ a day for two weeks and then $200 \mathrm{mg}$ taken three times a week (with at least $48 \mathrm{~h}$ between doses) for the next 22 weeks. In February 2016, it was announced that bedaquiline is to be made available in India and the drug will be available as part of second line treatment for patients suffering from MDR-TB and XDR-TB. The drug recently is under clinical trials phases in certain hospitals of the country. This drug was approved based on the phase IIb clinical trial results. However, phase III trials are still ongoing and its final approval is pending. There are issues with the safety concerns of bedaquiline, related to QTc prolongation caused by the drug and the

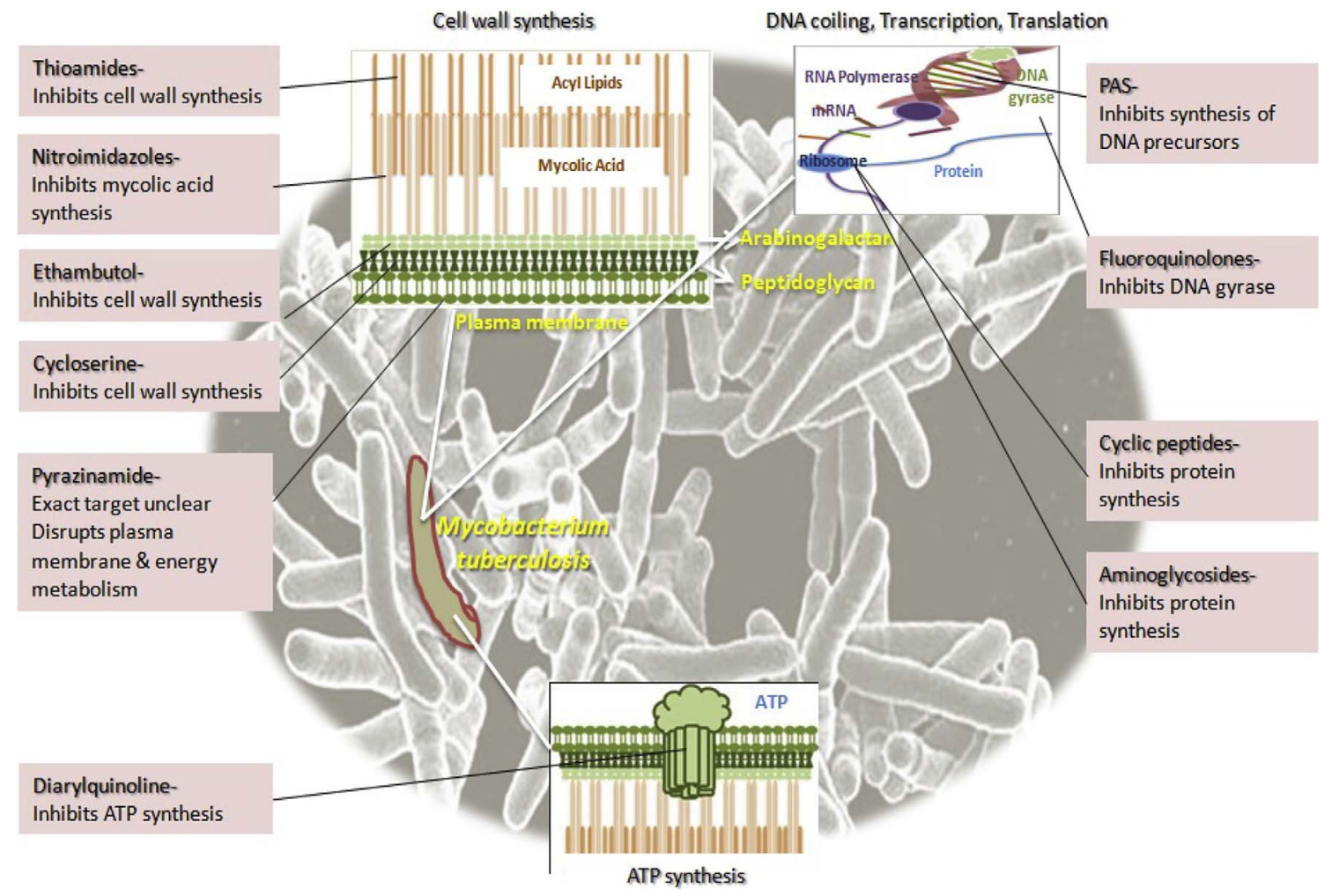

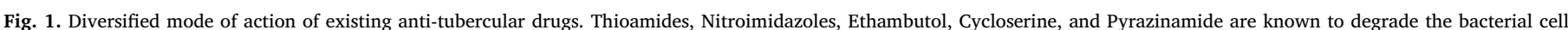

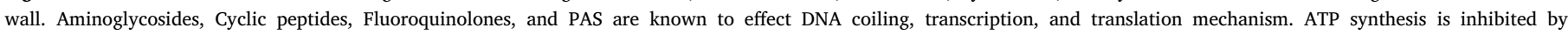
Diarylquinoline. 
Table 1

Commonly used anti-tubercular drugs, mechanism of action, and their mode of administration.

\begin{tabular}{|c|c|c|c|}
\hline Anti-tubercular drug & Mode of action & Mode of administration & References \\
\hline Bedaquiline & $\begin{array}{l}\text { Causes electroneutral uncoupling of respiration-driven ATP synthesis and blocks ATP synthase in } \\
\text { Mtb. } \\
\text { Binding and perturbing the } a-c \text { subunit interface of the } \mathrm{F}_{\mathrm{o}} \text {, corresponding to futile proton cycle } \\
\text { and show anti-tubercular activity. }\end{array}$ & Oral & [3] \\
\hline Capreomycin & $\begin{array}{l}\text { Protein synthesis inhibition by interrupting the interaction between ribosomal proteins L12 and } \\
\text { L10 }\end{array}$ & Intramuscular & [4] \\
\hline Cycloserine & $\begin{array}{l}\text { Interferes with early step bacterial cell wall formation inside the cytoplasm by competitive } \\
\text { inhibition of two enzymes, L-alanine racemase and D-alanylalanine synthetase. }\end{array}$ & Oral & [5] \\
\hline Ethambutol & $\begin{array}{l}\text { Disrupts arabinogalactan synthesis by blocking the arabinosyl transferase that leads to the } \\
\text { increased permeability of the cell wall. }\end{array}$ & Oral & [6] \\
\hline Isoniazid & $\begin{array}{l}\text { Blocking the natural enoyl-AcpM substrate and thus, inhibiting the fatty acid biosynthesis. } \\
\text { Finally it inhibits the mycolic acid synthesis. }\end{array}$ & Oral & [7] \\
\hline Kanamycin & $\begin{array}{l}\text { Inhibition of protein synthesis by irreversible binding to the cytosolic as well as membrane- } \\
\text { associated bacterial ribosome. }\end{array}$ & Intravenous & [8] \\
\hline Pyrazinamide & Mode of action not clear but thought to disrupt the membrane transport. & Oral & [9] \\
\hline $\begin{array}{l}\text { Rifampin, Rifabutin, Rifalazil and } \\
\text { Rifapentine }\end{array}$ & Protein synthesis inhibition as well as induction of programmed cell death. & Oral & [10] \\
\hline Nitroimidazoles & $\begin{array}{l}\text { Responsible for cell wall and DNA damage that causes DNA fragmentation, thereby inhibiting } \\
\text { DNA synthesis. }\end{array}$ & Oral & [11] \\
\hline AZD5847 & Impairment of the mycobacterial 50S ribosomal subunit. & Oral & [12] \\
\hline Fluoroquinolones & Blockage of DNA replication. & Intravenous & [13] \\
\hline Isoxyl & Inhibition of mycolic acids and fatty acids biosynthesis. & Pulmonary & [14] \\
\hline Ethionamide and Prothionamide & Inhibit the synthesis of mycoloic acids. & Oral & [15] \\
\hline Erythromycin and Roxithromycin & Inhibition of protein synthesis. & Oral & [16] \\
\hline p-aminosalicylic acid & Inhibition of thymine nucleotides biosynthetic pathways. & Oral & [17] \\
\hline Riminophenazines & Inhibition of electron transport chain. & Oral and parenteral & [18] \\
\hline Thiacetazone & Disruptive characteristics of the permeability of cell envelope and host immunomodulation. & Oral & [19] \\
\hline Delamanid & Inhibition of mycolic acid synthesis. & Oral & {$[20]$} \\
\hline Sutezolid & Prevents the initiation of protein synthesis in bacteria by binding to $23 \mathrm{~S}$ RNA. & Oral & [21] \\
\hline Streptomycin & Binds with 30 s subunit of the ribosome and interferes with protein synthesis. & Intramuscular & {$[22]$} \\
\hline Amikacin & Binds with the small subunit $30 \mathrm{~S}$ of the ribosome. & Intramuscular & {$[23]$} \\
\hline Ciprofloxacin & Targets the DNA gyrase. & Intravenous & {$[24]$} \\
\hline
\end{tabular}

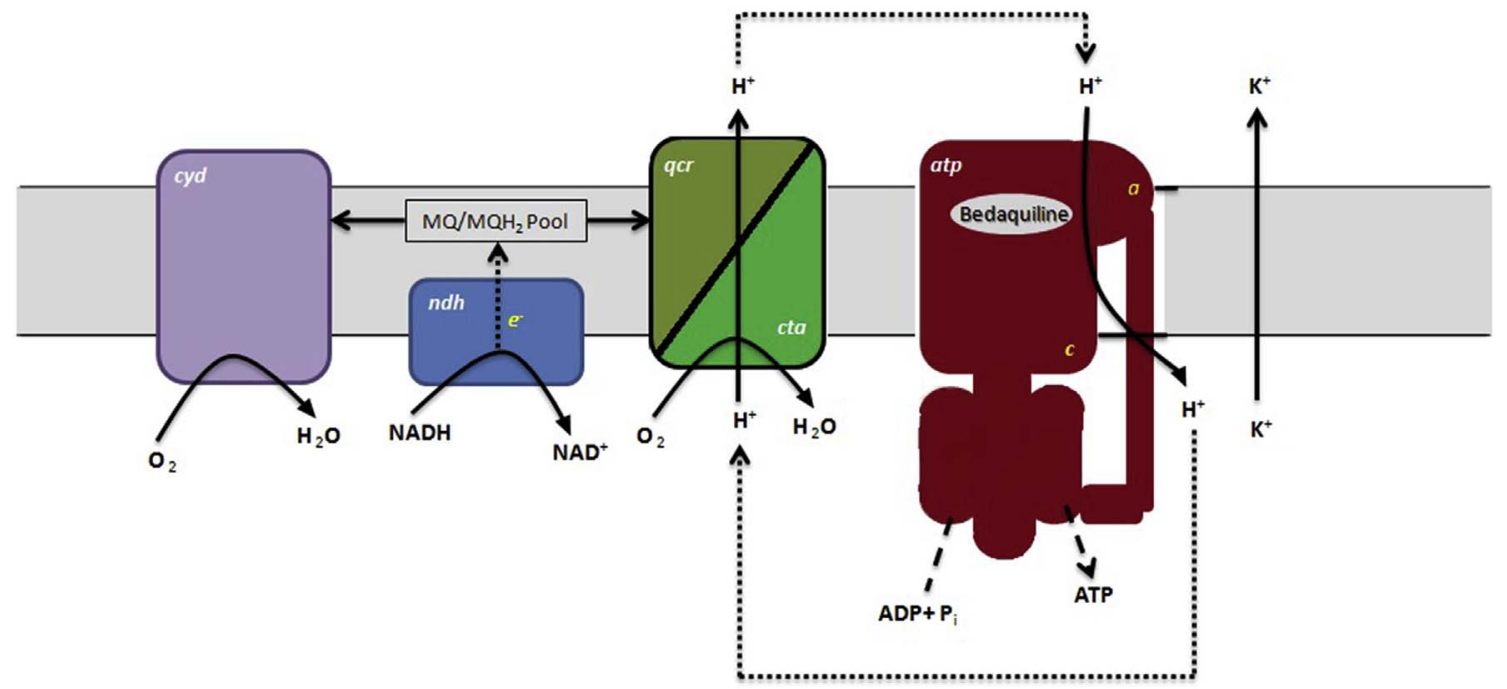

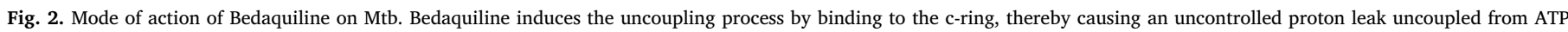
synthesis (adapted from [25]).

observed and yet unexplained higher mortality rate detected in the group treated with bedaquiline compared to placebo. However, smaller counts of patients showed lower mortality rate. A new phase III trial has begun in order to investigate the safety and efficacy of bedaquiline in combination with short MDR-TB regimens of 9 and 6 months duration, respectively.

Delamanid is another approved anti-tubercular drug, and the first drug of the nitroimidazole class to enter clinical practice. It is also known by its trade name of 'Deltyba'. It is currently being developed by the Otsuka pharmaceutical company as a treatment for MDR-TB. The drug is known to inhibit the synthesis of cell wall components of Mtb, particularly methoxy mycolic acid and ketomycolic acid. Delamanid is a pro-drug which gets activated by the enzyme deazaflavin dependent nitroreductase. A reactive intermediate metabolite, formed between delamanid and desnitro-imidazooxazole derivative, is considered to play a pivotal role in the blockage of mycolic acid production (Fig. 3). Similar to bedaquiline, a phase III trial on delamanid is ongoing. The trial includes the use of this drug in combination with moxifloxacin and HIV-positive patients on antiretroviral therapy (ART). In addition to this, other ongoing trials include assessing the use of delamanid for the treatment of pediatric MDR-TB. Preclinical and clinical phase results showed higher potency, lower risk for drug-drug interactions, and 


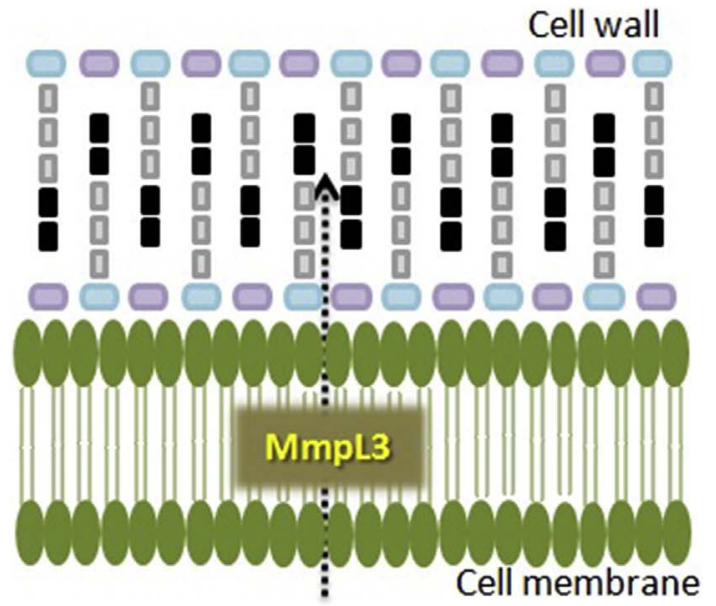

Mycolic Acid<smiles>CCCCC</smiles>

Mycolic Acid Biosynthesis

\section{Delamanid}

Fig. 3. Mode of action of Delamanid against Mtb. Delamanid inhibits the synthesis of cell wall components of Mtb, particularly methoxy mycolic acid and ketomycolic acid.

better tolerability of delamanid.

It is noteworthy that both bedaquiline and delamanid should only be prescribed as part of a WHO-recommended regimen for patients with MDR-TB in order to avoid the emergence of emergence of drug resistance Mtb. It is possible that both the drugs would be given to the same patient in the case of difficult-to-treat patients. One of the major challenges will be to ensure the availability of these drugs to all those in need while keeping in mind the WHO policies.

Apart from above mentioned anti-tubercular drugs, there are also few potent anti-tubercular agents in clinical trials status for the possible development of new therapeutic drugs against TB. These anti-tubercular agents are also known to inhibit the growth of Mtb through various mechanism of action as shown in Fig. 4. Most of them are in preclinical development (40\%), followed by Phase II (35\%) and Phase III $(25 \%)$ phases. Of the new anti-tubercular agents under clinical trials, only 5 are in Phase III and 7 are in Phase II trials. Additionally, 8 antitubercular agents are under preclinical development phage, including 3 antimycobacterial agents in Good Laboratory Practice toxicity evaluation. At present, there are no anti-tubercular drugs registered in Phase I. Drugs under preclinical development may enter into Phase I in future. Khusro and Aarti [26] reviewed the pivotal role of TB-PACTS (TBPlatform for Aggregation of Clinical TB Studies) in making clinical trials data of TB easily available. Authors further mentioned that TB-PACTS is an invaluable data sharing approach in order to combat TB. In fact, it is designed to catalyze and step up TB research by curating and standardizing Phase III TB clinical trials data and making those data easily available to the scientific community.

\section{A glance at current WHO report of TB}

According to the latest report of WHO [27], there are increased total counts for new TB cases globally than in last few years. In spite of the recent advances and the fact for curing $\mathrm{TB}$, the disease remains one of the world's deadliest threats. The medications for the treatment of TB can also lead to other serious health issues, such as kidney failure, liver, or heart disorder, loss of vision or hearing, psychosis, and most importantly HIV infection. Worldwide, $12 \%$ of the new TB cases are found to be HIV-positive. In fact, HIV infection impels the risk of TB infection on exposure, progression from latent infection to active, and finally a risk of mortality if not treated both TB and HIV in time. Correspondingly, TB is the most common opportunistic infectious disease and cause of mortality among HIV infected persons.

The desideratum aim of WHO report 2017 is to provide a comprehensive and detail assessment of the TB epidemic and progress in diagnosis, prevention, and treatment globally. The report also targets a $90 \%$ reduction in the TB incidence rate (Fig. 5a) and 95\% reduction in TB deaths (Fig. 5b) in 2035, compared with levels in 2015 (per 100000 populations) in order to achieve End TB strategy. The 2030 targets are an $80 \%$ reduction in the TB incidence rate and a $90 \%$ reduction in TB deaths, compared with levels in 2015. The TB report 2017 establishes an essential landmark worldwide in TB monitoring by WHO. There are few important highlights of WHO 2017 reports as listed below.

- In 2016, there were an estimated 1.3 million TB deaths among HIVnegative people (down from 1.7 million in 2000) and an additional 374000 deaths among HIV-positive people.

- An estimated 10.4 million people were infected with TB in 2016: $90 \%$ were adults, $65 \%$ were male, $10 \%$ were people living with HIV (74\% in Africa), and 56\% were in five countries: India, Indonesia, China, the Philippines, and Pakistan.

- In 2016, there were 600000 new cases with resistance to rifampicin, the most effective first-line drug, of which 490000 had MDR-TB. Almost half $(47 \%)$ of these cases were in India, China, and the Russian Federation.

- In 2016, 6.3 million new cases of TB were reported (up from 6.1 million in 2015), equivalent to $61 \%$ of the estimated incidence of 10.4 million; the latest treatment outcome data show a global treatment success rate of $83 \%$.

- There were 476774 reported cases of HIV-positive TB ( $46 \%$ of the estimated incidence), of whom $85 \%$ were on ART.

- A total of 129689 people were started on treatment for drug-resistant TB, a small increase from 125629 in 2015 but only $22 \%$ of the estimated incidence; treatment success remains low, at $54 \%$ globally.

- Ten countries accounted for $76 \%$ of the total gap between TB incidence and reported cases; the top three were India (25\%), Indonesia (16\%), and Nigeria (8\%).

- Most of the estimated number of incident cases in 2016 occurred in the WHO South-East Asia Region (45\%), the WHO African Region (25\%), and the WHO Western Pacific Region (17\%); smaller proportions of cases occurred in the WHO Eastern Mediterranean Region (7\%), the WHO European Region (3\%), and the WHO Region of the Americas (3\%).

- The annual number of incident TB cases relative to population size varied widely among countries in 2016, from under 10 per 100000 populations in most high-income countries to $150-300$ in most of the 30 high TB burden countries, and above 500 in a few countries including the Democratic People's Republic of Korea, Lesotho, Mozambique, the Philippines, and South Africa.

- Globally in 2016, 57\% of notified TB patients had a documented HIV test result, up from 55\% in 2015.

- The treatment success rate for HIV-associated TB (2015 cohort) was $78 \%$ and for XDR-TB (2014 cohort) it was 30\%.

- At least 35 countries have introduced shorter regimens for treatment of MDR-TB. As part of efforts to improve outcomes for MDR/XDR$\mathrm{TB}, 89$ countries and territories had started using bedaquiline and 54 had used delamanid by June 2017. 


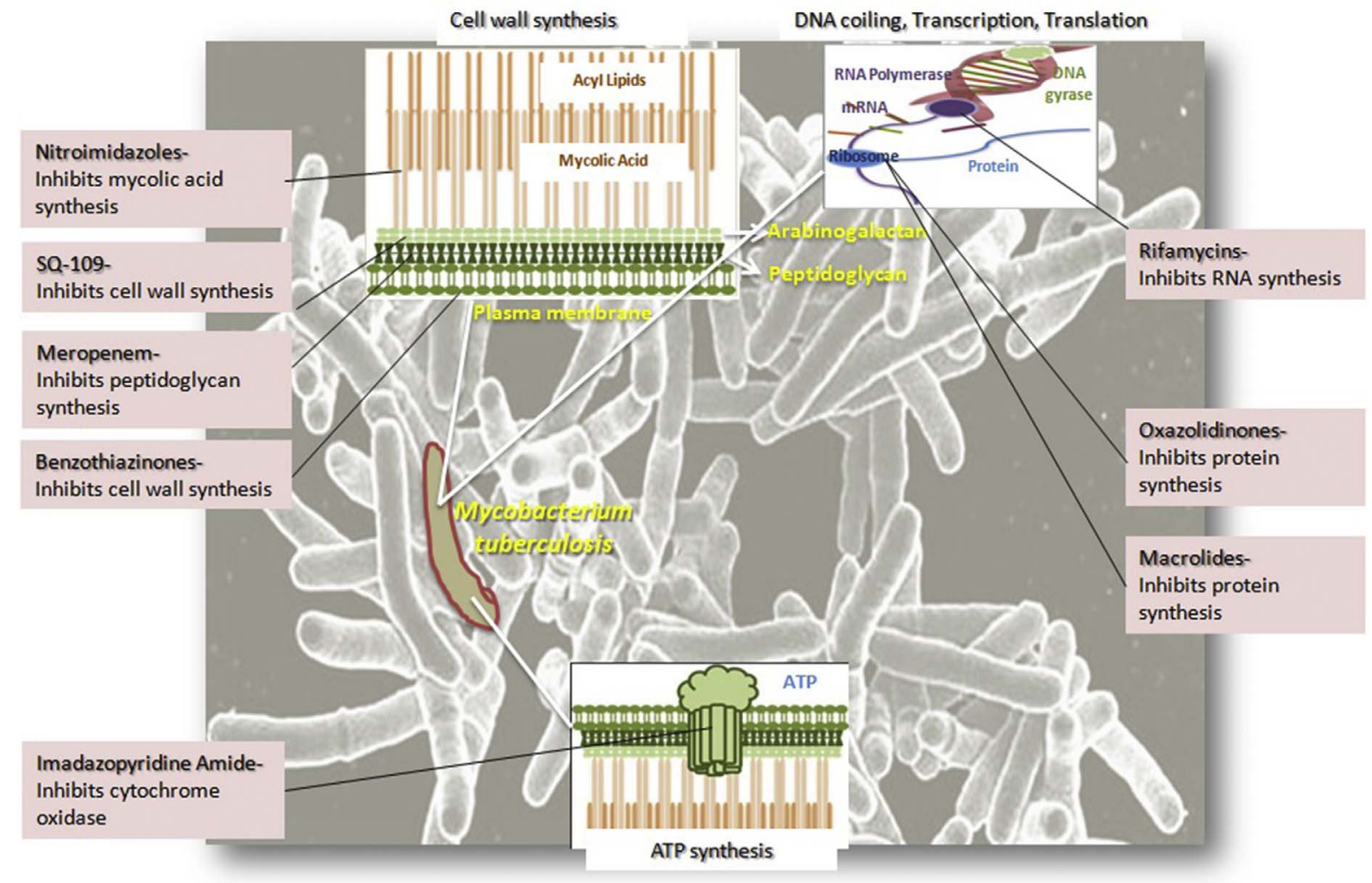

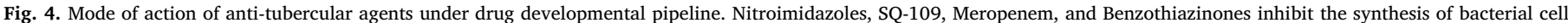

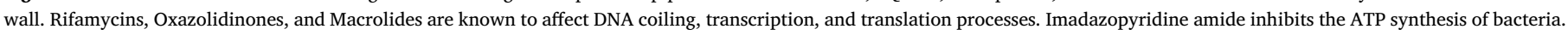
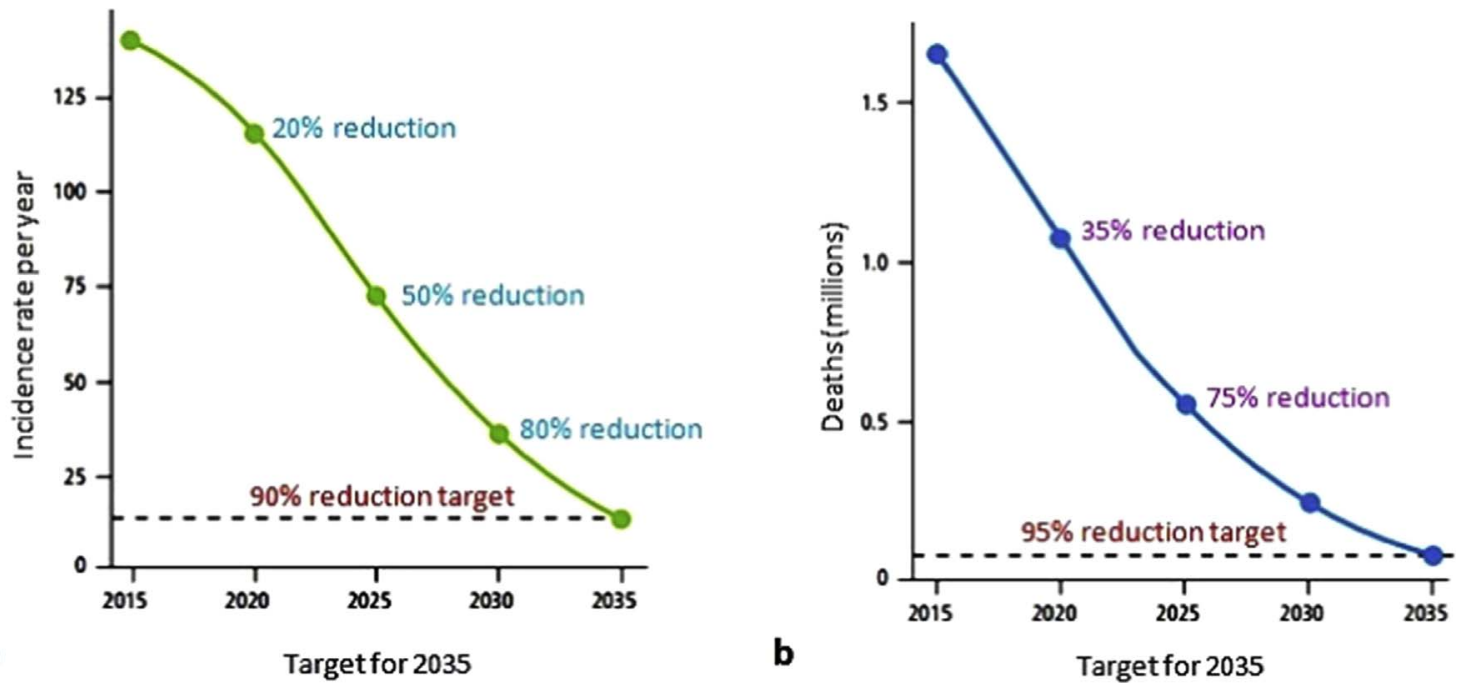

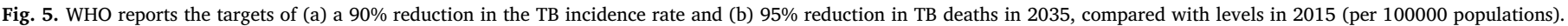

- Funding for TB care and prevention reached US\$ 6.9 billion in 2017 in 118 low and middle-income countries. This was an increase from US\$ 6.3 billion in 2016 and more than doubles the US\$ 3.3 billion that was available in 2006.

- India stood out as a country in which the budget envelope for TB was substantially increased in 2017.

- Of the 10.4 million incident cases of TB in 2016, an estimated 1.9 million were attributable to undernourishment, 1.0 million to HIV infection, 0.8 million to smoking, and 0.8 million to diabetes.

- There are 17 drugs in Phase I, II or III trials, including eight new compounds, two drugs that have received accelerated or conditional regulatory approval based on Phase IIb results, and seven repurposed drugs. Various new combination regimens are in Phase II or Phase III trials.

- There are 12 vaccine candidates in clinical trials: three in Phase I, and nine in Phase II or Phase III.

- TB incidence is falling at about $2 \%$ per year and $16 \%$ of TB cases die from the disease; by 2020 , these figures need to improve to $4-5 \%$ per year and $10 \%$, respectively, to reach the first (2020) milestones of the End TB Strategy. 


\section{Anti-tubercular/antimicrobial peptides and molecular mode of action}

Anti-tubercular peptides are naturally synthesized endogenous oligopeptides containing different numbers of amino acids that shows plausible antimycobacterium activity. In fact, anti-tubercular/antimicrobial peptides belong to gene-encoded peptides that show direct growth inhibitory activity against several Mycobacterium species, especially Mtb and devote a key appearance in host defence mechanism. Development of MDR- and XDR-Mtb has heightened the imperative to understand the mode of operation of peptides in response to bacterial targets. Generally, cationic antimicrobial peptides (AMPs) interact with the anionic surface of bacterial membrane, thereby causing the conglomeration of peptide molecules on the membrane. A specific peptide-lipid complex is formed after the hydrophobic interaction between the AMPs and the bacterial membrane, thereby producing the possible alteration in the structure of bacterial membrane [28]. The modification favours the translocation of peptides across the cell membrane and targets the intracellular components such as enzymes, nucleic acids, and other organelles by diffusing into the cytoplasm.

The barrel-stave model, the toroidal model, and the carpet model are commonly acknowledged for killing the bacteria (Fig. 6). In the barrel-stave model, the peptides arrange themselves in order to bind to the cell membranes, thereby causing the transformation of the peptide into a bilayer. Therefore, the hydrophobic peptides are arranged in line with the lipid centre, while the hydrophilic peptides create an access pore in the internal section of the membrane. The carpet model involves disruption of the membrane through peptide binding to the external layer of the cell membrane, thereby creating a carpet. In the toroidal model, the attached peptides begin to compel the lipid monolayer to constantly bend through the pores. Thus, the core is lined by the inserted peptides as well as lipid head groups [29,30].

Previous report has demonstrated the antimycobacterial activity of peptides by increasing the permeability of bacterial cell wall [31]. The distinctive cell wall composition of Mtb contributes to its pathogenicity nature and acts as an impediment to the ingress of anti-tubercular peptides/drugs. In fact, the bacterial cell surface is hydrophobic due to the high content of lipid and mycolic acids with long hydrocarbon chains. This extremely unique characteristic of Mtb induces the aggregation of the cells and consequently affects the permeability of the cell wall [32]. AMPs could also incite the cell membrane permeability not only by creating pores but also disrupting the mechanisms of cell wall synthesis. There are also few reports discussing the AMPs with more than one mode of anti-tubercular action. Carroll et al. [33] demonstrated that lantibiotics such as nisin and lacticin 3147 bind to the peptidoglycan precursor and thus, altering the biosynthesis of peptidoglycan as well as creating pores in the cell membrane. Uridyl-peptide antibiotics might inhibit bacterial translocase MraY and exhibit antimycobacterial property. The growth inhibitory characteristic of mycobacterium with similar mode of action was reported by Li et al. [34].

\section{Physiochemical parameters for antimicrobial peptides}

Amphipathicity nature of peptides is an imperative physiochemical factors required for their growth inhibitory action against the bacteria. In general, amphipathicity ensures the commencing interaction between peptides and bacterial membrane, and thereby helps the binding

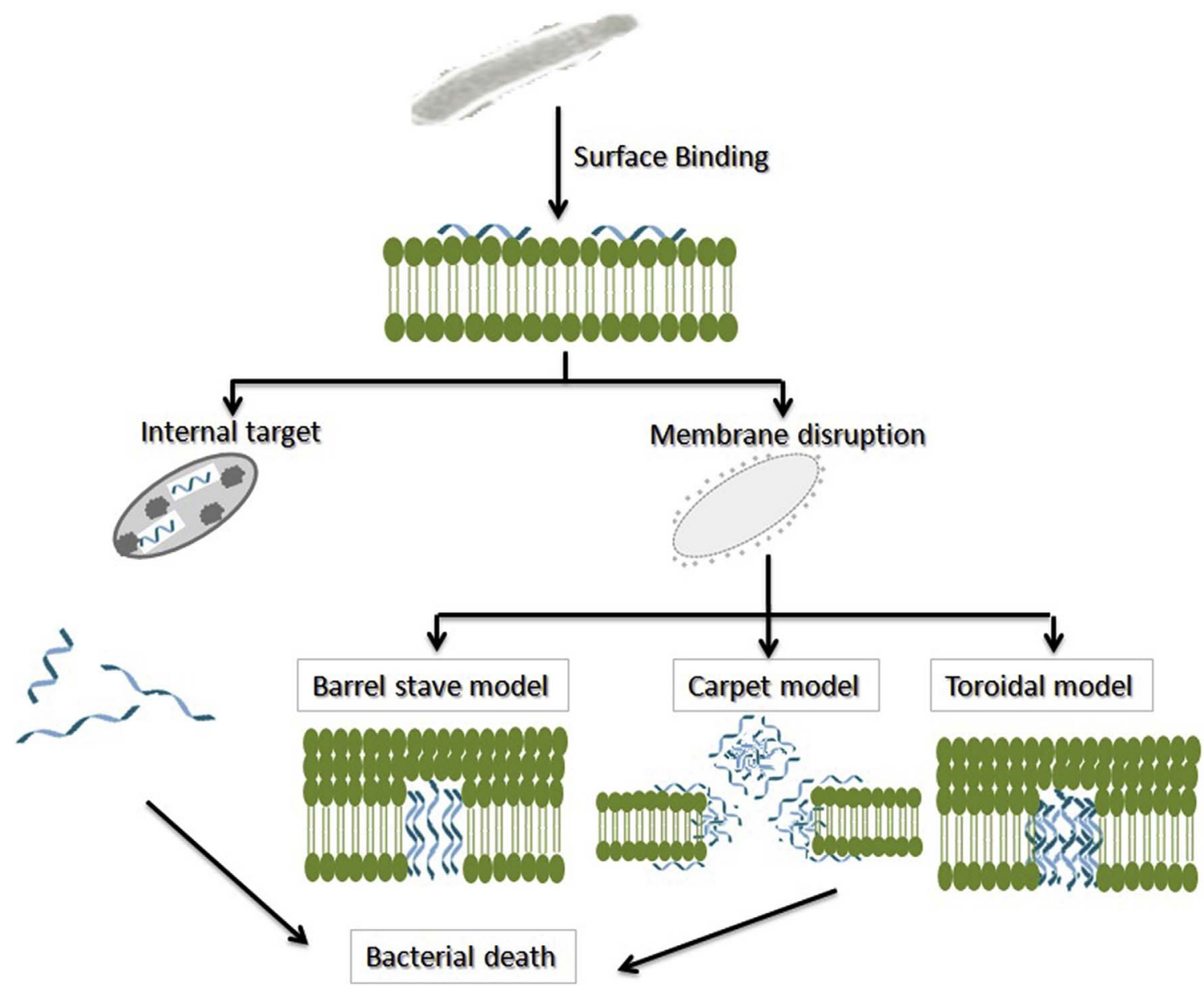

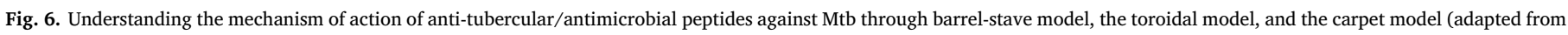
[30]). 
of peptides with membrane surface. Almost all peptides form amphipathic structures upon interaction with target bacterial membranes. The disulphide bonds existing in the structure of peptides stabilize and induce the antimycobacterial activity [35].

Hydrophobicity is another important parameter influencing the bioactivity of peptides. Peptide hydrophobicity is the percentage of hydrophobic residues within a peptide and hydrophobicity administers the intensity to which a peptide can partition into the lipid bilayer. According to the report, the efficiency of antimycobacterial activity is intensified due to the balance between the hydrophobicity of selective peptide and its polar regions [36]. An increase in the hydrophobic moment results in a significant increment in the permeabilizing properties of peptides against bacterial membranes. Previous reports demonstrated that an increase in the hydrophobicity on the positively charged side of a peptide below a threshold range can induce its activity, while decreasing hydrophobicity might have negative impact on the bioactivity of peptides [37], demonstrating an optimal hydrophobicity for selective peptide, beyond which its activity subsides [38]. Therefore, the hydrophobicity under optimum range will help to design synthetic peptides dexterously.

The length of a peptide plays an exceptional role in designing a new synthetic peptide. A minimum of 7-8 amino acids are required to form amphipathic structures with hydrophobic and hydrophilic regions on the reverse sides of a peptide. The size for a peptide to transverse the lipid bilayer of the bacterium in the barrel-stave model should be noticeably 22 and 8 amino acids for $\alpha$-helical and $\beta$-sheet peptides respectively. Generally, a peptide with shorter residues exhibit less toxicity that contributes in the development of ultramodern synthetic peptides.

The net charge of selective peptides is one of the leading aspects for the initial interaction with negatively charged bacterial membranes. The bioactivity of peptides can be induced by altering the net charge of the peptide without any side effects on the host. Similarly, solubility of peptides is a major framework because peptides should ingress through lipid membranes, and thus they need to be soluble in aqueous environments.

Apart from the above mentioned physiochemical parameters, it is obligatory to find out if a candidate peptide is toxic for host cells. The cytotoxicity activities of peptides are not exercised to its anti-tubercular activity due to the difference in the structural property of candidate peptide [39]. The study has demonstrated that enniatins from fungal genera such as Alternaria, Fusarium, Halosarpheia, and Verticillium evoked toxicity using in vitro assays [40]. Sharma et al. [41] reported a reduced toxicity of HNP-1 towards the macrophage cell line at a concentration inhibitory to mycobacterium, thereby suggesting the low toxicity because of the presence of FBS in the culture media. It is highly recommended to find new anti-tubercular peptides with reduced or null toxicity to human cells.

\section{Anti-tubercular peptides and Mtb: A computational approach}

Novel drug targets identification depends upon the identification of essential genes present in the bacterium. The genome sequence of pathogenic bacteria provides a tremendous amount of information that can be utilized in drug target identification against pathogen with respect to the host. The application of computational tools such as molecular dynamics, docking, and modelling are often being used in order to understand the protein complexes involved in mycobacterium infections. Computational approaches or in silico studies provide opportunities to find the potential drug targets from proteome analysis. At present, the computational approach has replaced the experimental tools in order to understand the physicochemical and structural properties of proteins. Proteins are the building block and are located in the sub-cellular compartment such as outer membrane, the inner membrane, periplasm, cytoplasm, and extracellular of prokaryotes. The identification of various sub-cellular proteins contributes in designing and developing novel anti-tubercular drugs. The structural modelling of these proteins can help to determine which of these proteins can function as most effective surface epitope for the drug that can lead to the discovery of novel therapeutic anti-tubercular drug. On the other hand amino acid sequences determine and characterize molecule's functional, physical, and chemical properties.

Host-pathogen protein interactions (HPIs) play a versatile role in the pathogen's ability to invade the host and escaping from host's immune defences. Experimentally, the yeast two-hybrid (Y2H) system, luminescence-based mammalian interactome mapping, and co-immunoprecipitation (coIP) coupled with mass spectrometry (MS) are main approaches to detect the interacting proteins [42]. But these techniques are not only time-consuming but also an expensive approach. Hence, computational methods have been considered to identify the protein-protein interaction (PPI) that is based on protein sequences, structural, and genomic features. Interologs (homologous PPI) is based on the assumption that homologous proteins preserve their potential to interact [43] and detect host-pathogen protein interactions [44].

At present, the complete genome of Mtb and the host Homo sapiens has been sequenced that insights the prediction of host-bacteria protein interaction. It helps to compile a list of potential gene products and identify the functions present in the human and absent in Mtb. It has solved the major problem of searching for potential drug targets. The anti-tubercular peptides are also known for inhibiting the bacterial enzymes and therefore, these enzymes can be drug targets. There are several pathways that can be targeted for rational drug design. Few targets such as thiamine metabolism, peptidoglycan biosynthesis, polyketide sugar unit biosynthesis, D-Alanine metabolism, fatty acid biosynthesis path1, fatty acid metabolism, phospholipid degradation, glycerolipid metabolism, ubiquinone and menaquinone biosynthesis, lysine biosynthesis, pyrimidine metabolism, purine metabolism, nitrogen metabolism, nucleotide sugar metabolism, inositol phosphate metabolism, and glycerolipid metabolism had been recommended for designing a drug against Mtb [45].

Glutamine synthetase is an important enzyme that produces the condensation reaction along with glutamate [46]. It is considered as a central part of the bacterial nitrogen mechanism and catalyzes the glutamate. It leads to the formation of glutamine and hydrolysis of ATP. This enzyme is available in the brain and kidney. In the brain, it binds with glutamate regulation that leads to the detoxification of ammonia, and finally terminates the neurotransmitter signals. Tryptophan, histidine, alanine, glycine etc. can inhibit glutamine synthetase. This enzyme is an essential target for maximum number of anti-tubercular drugs.

RNA polymerase is yet another crucial enzyme that catalyzes the synthesis from a DNA template [47]. It is also called as DNA-dependent RNA polymerase. The enzyme is very essential for the growth and survival of the mycobacterium. This intracellularly targeted enzyme is known for the transcription and termination of DNA sequence. Perumal et al. [48] performed the molecular docking studies of short peptides related to human histatin and apidaecin intolerant asthma against glutamine synthetase and RNA polymerase respectively. Authors also compared the anti-tubercular potential of peptides with reference to the standard anti-TB drug such as rifampicin, isoniazid, amikacin, and ciprofloxacin. According to their reports, aspirin-intolerant asthma (AIA)II and AIA-I showed activity against the glutamine synthetase with GLIDE score ( $\mathrm{G}$ score) of -8.79 and -7.97 as compared to standard amikacin and ciprofloxacin with $\mathrm{G}$ score of -7.71 and -3.45 . On the other hand, AIA-I, AIA-II, and HH-I showed potency against RNA polymerase with $\mathrm{G}$ score of $-10.12,-9.47$, and -8.74 in comparison to rifampicin and isoniazid with $\mathrm{G}$ score of -6.57 and -3.34 . The findings proved that the docked aspirin-intolerant asthma (AIA)-II and AIA-I could be utilized for developing new chemical entity against Mtb. The G score can be applied as a semi-quantitative descriptor and confirmed that above inhibitors are suitable as the binding pocket of the 
glutamine synthetase and RNA polymerase receptors. Based on their outcomes, the authors recommended exploring the novel peptides as anti-tubercular agents.

Menaquinone is a lipid-soluble naphthoquinone that is necessary for various pivotal functions of bacteria and it serves as an electron transporter in the respiratory chain for their survival strategy. The chorismate of the shikimate pathway in bacteria induces the synthesis of naphthoquinone. The menaquinone has been an attractive target for the development of potential drug against Mtb due to its absence in humans and animals.

$O$-succinylbenzoate synthase (OSBS) plays a pivotal role and it is one of the major potential drug targets in shikimate pathway. In spite of the availability of several anti-tubercular drugs, this enzyme has been taken into account due to the emergence of MDR-TB and XDR-TB. Mtb$O$-succinylbenzoate synthase (Mtb-OSBS) converts 2-succinyl-6-hydroxy-2,4-cyclohexadiene-1-carboxylate (SHCHC) into 4-(2'-carboxyphenyl)-4-oxobutyrate (O-succinylbenzoate or OSBC or menC) via an elimination of water. Pulaganti et al. [45] developed first time the 3-D structure of Mtb-OSBS followed by its structural annotations. The 3-D model of Mtb-OSBS was built using structure coordinates of Thermobifida fusca. Further, substrate-based analogue and auxiliary molecule interaction energies with Mtb-OSBS were systematically evaluated using molecular dynamic simulation and molecular docking strategies in order to develop active drugs against Mtb-OSBS. The active site amino acids had been identified by comparing the template sequence with the Mtb-OSBS sequence. The authors identified that Lys ${ }^{108}, \mathrm{Asn}^{140}$, $\mathrm{Asp}^{138}, \mathrm{Lys}^{110}, \mathrm{Glu}^{189}, \mathrm{Ser}^{236}, \mathrm{Asp}^{188}, \mathrm{Arg}^{27}, \mathrm{Tyr}^{52}$, and $\mathrm{Ser}^{237}$ are highly conserved, and these may contribute as active residues. Authors screened 2-succinyl-6-hydroxy-2,4-cyclohexadiene-1carboxylate (SHCHC) through AutoDock 4.0. and the SHCHC molecule was further modified structurally and optimized through PRODRG server. MD simulation showed the stable conformation annotations of Mtb-OSBS and proved hypothetically to develop better anti-tubercular drugs.

In the line of exploring computational tools to design and developing novel drugs against Mtb, Huo et al. [49] developed a systematic flow to predict the interaction between Mtb and H. sapiens based upon sequence motifs. Protein sequences were used as an initial input to identify the interaction between Mtb and H. sapiens by 'interolog' method and further filtered by domain-domain interactions (DDIs) prediction. As a result of this, 118 pairs of host-pathogen interactions were identified, constituting 43 proteins from Mtb and 48 proteins from H. sapiens. The computational tools such as VisANT, Reactome, InteroPorc, IntAct, DIP, MPIDB, MINT, and HPRD were applied to predict intra-species PPIs for the proteins from Mtb and H. sapiens [50-57].

A new specialized database named PATH (Protein interactions of Mtb and Human) was constructed in order to store those predicted interactions and proteins. A total of 118 credible HPIs were identified and stored in the PATH. The database supports the search related to the interspecific and intraspecific interactions between MTb and human, MTb's gene locus, Uniprot ID, and might facilitate the understanding of mechanism behind the TB infection. The PATH database may help to develop new anti-tubercular drugs and may shed light on the interaction between Mtb and its host. In fact, PATH was built on an Nginx with Python and a MySQL Server as the back-end. Hyper Text Markup Language (HTML), JQuery, and Cascading Style Sheets (CSS) were applied at the front-end.

Generally, the selection of suitable antimicrobial peptides against the target pathogens depends upon the several parameters. The Isoelectric point (pI) determines the solubility and the mobility of peptide or protein of interest. Computed pI values of proteins greater than 7 indicate the basic nature of protein. The isoelectric point is useful to develop a buffer system for the purification by isoelectric focusing method and also to determine half life of protein [58]. The instability index determination is another parameter for the selection of suitable peptides. The protein with instability index value lower than
40 is predicted as stable, while greater than 40 is considered as unstable. The grand average hydropathy (GRAVY) value for a peptide is calculated by dividing the sum of hydropathy values of all amino acids to the number of residues in the sequence. The lower GRAVY range indicates the hydrophilic nature of the peptides. SOSUI predicts the transmembrane helices for soluble proteins. Self Optimized Prediction Method with Alignment (SOPMA) predicts the secondary structures of proteins. The secondary structure demonstrates the helical, strand or coil nature of the given amino acids. The three homology modelling programs, Swiss Model server, Modeller, and EsyPred3D web Server help to model the 3-D structure of the peptides or proteins of interest and contribute in the selection of the best model. The lower $\mathrm{E}_{\min }$ value predicts the suitability of peptides of interest for designing therapeutic drugs.

In response to the above mentioned parameters for designing therapeutic drugs from antimicrobial peptides, Bhagat et al. [58] performed docking study with antimicrobial peptide, aurein 1.2 along with available drugs such as pyrazinamide, ethambutol and isoniazid against three proteins (NP_216679, NP_218309, NP_218312) of Mtb as potential targets. Among these proteins chosen, NP_218312 was found to be the most stable structure with good stereo-chemical properties, thereby making this protein of interest as an effective target. Aurein1.2 was found to be the most potent anti-tubercular peptide than other drugs investigated and was recommended for designing an emphatic drug against Mtb.

The cytosolic Fau protein (a microbicidal peptide precursor) is composed of ubiquitin-like domain FUBI at N-terminus and ribosomal protein rpS30 at C-terminus. Fau has been important in killing of intracellular Mtb infection through autophagy-targeting p62 mechanism. The p62 adaptor protein delivered rpS30 to autolysosome where it was converted into antimicrobial peptides capable of killing Mtb in mycobacterial phagosome. Autophagy is a homeostatic process of eukaryotic cells that exposes cytoplasmic components, proteins, organelles, and various intracellular pathogens to lysosomes for their digestion. The autophagy degrades intracellular microbial pathogens by two principal methods. First, autophagosomes directly engulf free cytosol microorganisms that marked with poly-ubiquitin complexes through adaptor protein such as p62 and NDP52 [59], and then delivered for its elimination. Second, autophagy exposes Fau protein (a precursor of rpS30 peptides which was converted into microbicidal ribosomal protein by proteolytic enzyme in autolysosome) to the phagosome of Mtb through p62 autophagy adaptor proteins. The UBA domain of p62 proteins directly bind with Fau, as indicated by immunofluorescence microscopy and immunoprecipitation.

FUBI had ability to interact to the p62 UBA domain directly [60]. FUBI and p62 UBA domain are members of protein family UBL (ubiquitin-like) and UBD (ubiquitin-binding domain), respectively, and binding interactions between UBL and UBD have been reported [61]. In this regard, Chongruchiroj et al. [62] elucidated the interaction between FUBI and p62 UBA. Based upon the conserved hydrophobic regions of FUBI and p62 UBA domain, a total of 334 docked poses were predicted. A plausible structure of FUBI in complex with p62 UBA domain was obtained by several computational tools such as ZDOCK, RDOCK, MD simulations, and MM-PBSA binding free energy calculation. Based on this, five possible binding modes were illustrated with different orientations of p62 UBA domain with respect to FUBI. However, only two binding mode structures were found to be stable after 15 ns of MD simulations. On the other hand, their MM-PBSA binding energies were analyzed to demonstrate the potent FUBI-p62 UBA complex structure. Thus, the report enlightened the interaction between p62 and Fau which is a very prominent step of autophagy-targeting p62 mechanism for the growth inhibition of Mtb.

The presence of mycolic acid in the outer membrane is an important criterion for the existence and pathogenicity of Mtb. In fact, mycolic acids are hydroxyl fatty acids containing long alkyl side chains that allow the bacterium to condone and sustain inside the macrophages by 
protecting it against host's immune systems. The bacterium secretes mycolyl transferases with molecular weight ranging from $30 \mathrm{kDa}$ to $35 \mathrm{kDa}$, called as antigen-85 protein complexes (Ag85A, Ag85B, and Ag85C). They are mainly responsible for the synthesis of mycobacterial cell wall. Mycolyl transferase catalyzes the transfer of mycolic acid and induces the synthesis of bacterial cell wall. In view of the pivotal role of mycolyl transferase in the synthesis of bacterial cell wall, they are inferred as an ideal target for the TB treatment. In general, these bioactive proteins catalyze the transfer of mycolic acid from one trehalose 6monomycolate (TMM) to another, and forms trehalose 6,6-dimycolate (DMM) as well as free trehalose.

Dermcidin is one of the antimicrobial peptides which is encoded by the $D C D$ gene in human being and often secreted by sweat gland cells to provide a broad spectrum antibacterial activity against several pathogens. From this point of view, Banerjee and Gohil [63] demonstrated the binding interaction between the dermcidin and mycolyl transferase using AutoDock Vina, a computational docking strategy. They concluded and postulated that dermcidin has the ability to interact with FbpA (target for ligand binding) in order to inhibit the transfer of mycolic acid to the peptidoglycan layer of the bacterial cell wall and ultimately interfering with the synthesis of cell wall. According to the report, the Global Model Quality Estimation (GMQE) score for the modelled protein was found to be 0.98 , which indicated the accuracy of the model and the docked model was visualized using PyMol. In addition to this, the study also reported the lowest energy value $(5.6 \mathrm{kcal} /$ mol) relative to previous reports, and therefore, the model was considered the best fit.

Toxin-antitoxin (TA) systems contribute in multidrug tolerance and biofilm formation properties of bacteria. TA systems have been proved to be targets to develop offbeat anti-tubercular agents [64] because the bactericidal agents disrupt the interaction of the TA complex that may lead cells to death [65]. The VapBC family (47 of 88 putative TA systems) constitutes more than half of the TA systems in Mtb [66], and vapBC loci are responsible for bacterial persistence and pathogenicity factors [67]. In order to develop novel anti-tubercular agent, Lee et al. [68] concentrated on VapBC Systems of Mtb. The finding reported that Mtb VapC30 toxin maintained the growth through both the magnesium and manganese ion-dependent ribonuclease activity and was inhibited by the cognate VapB30 antitoxin. The study led to design VapB30 and VapC30-associated new peptides which successfully disrupted the toxin-antitoxin complex and thus activated the ribonuclease activity of the VapC30 toxin. The study reported the designed peptides that mimic the helical regions of the toxins in the heterodimer interface of the VapBC30 complex, and disrupt the binding between VapBC30 complexes. The approach may prove to be a potential candidate in TB treatment. The VapBC5, VapBC3, and VapBC15 complexes are the major types of Mtb VapBC family members [69] whose structural information and functional mechanisms are still elusive.

Keeping in view of the previous in silico studies, docking strategy proved to be an effective approach for the designing and developing new anti-tubercular drugs from the peptides. This novel approach paves a new way for worldwide researchers to investigate further the researches in depth, based on the data obtained through computational studies. The systematic computational analysis conducted in the previous reports may contribute in designing better drugs for the treatment of TB. The computer-aided tools put forth a foundation for screening several anti-tubercular peptides from diverse sources in order to develop novel drugs for the TB therapy.

\section{Concluding remarks and future perspectives}

TB is a global endemic disease that has become obligatory to treat due to the emergence of MDR- and XDR-Mtb. Currently available frontline anti-tubercular drugs are failing to prevent spread of the disease and causing the development of resistant strains due to the unique pathogenicity of Mtb. WHO reported that new drugs will be required to achieve the targets set in the End TB strategy. Anti-tubercular peptides from diverse sources might be a suitable and effective alternatives of existing antimycobacterium drugs due to their broad spectrum activity against Mtb. Studying the systematic computational analysis between peptide structure and its molecular mode of action will lead to design and develop novel drugs with desired mycobactericidal activities that may create a new era for the possible eradication of $\mathrm{TB}$ in future.

\section{Conflicts of interest}

We declare that we have no conflict of interest.

\section{References}

[1] WHO, Global tuberculosis report 2012, WHO, Geneva, Switzerland, 2012.

[2] J.G. Rodriguez Plaza, B. Rivas-Santiago, R. Hernandez-Pando, G.D. Rio, Prospective Tuberculosis treatment: peptides, immunity and autophagy, J. Mol. Genet. Med. 8 (2014) 128, http://dx.doi.org/10.4172/1747-0862.1000128.

[3] K. Hards, J.R. Robson, M. Berney, L. Shaw, D. Bald, A. Koul, et al., Bactericidal mode of action of bedaquiline, J. Antimicrob. Chemother. 70 (2015) 2028-2037.

[4] Y. Lin, Y. Li, N. Zhu, Y. Han, W. Jiang, Y. Wang, et al., The antituberculosis antibiotic Capreomycin inhibits protein synthesis by disrupting interaction between ribosomal proteins L12 and L10, Antimicrob. Agents Chemother. 58 (2014) 2038-2044.

[5] Y.H. Lee, J.D. Helmann, Reducing the level of undecaprenyl pyrophosphate synthase has complex effects on susceptibility to cell wall antibiotics, Antimicrob. Agents Chemother. 57 (2013) 4267-4275.

[6] H. Li, A. Cowie, J.A. Johnson, D. Webster, C.J. Martyniuk, C.A. Gray, Determining the mode of action of anti-mycobacterial C17 diyne natural products using expression profiling: evidence for fatty acid biosynthesis inhibition, BMC Genomics 17 (2016) 621, http://dx.doi.org/10.1186/s12864-016-2949-y.

[7] A.M. Elhagi, A.R.N. Ben Naji, S.M. Bensaber, T.K. Almog, Microwaves assistant technique in spectrophotometric assay of isoniazid using it's schiff's base derivatives, Int. J. Pharm. Sci. Res. 4 (2013) 644-649.

[8] D.T. Hoagland, J. Liu, R.B. Lee, R.E. Lee, New agents for the treatment of drug resistant Mycobacterium tuberculosis, Adv. Drug Deliv. Rev. 102 (2016) 55-72.

[9] S.T. Pullan, J.C. Allnutt, R. Devine, K.A. Hatch, R.E. Jeevas, C.L. Hendon-Dunn, et al., The effect of growth rate on pyrazinamide activity in Mycobacterium tuberculosis - insights for early bactericidal activity? BMC Infect, Dis 16 (2016) 205, http://dx.doi.org/10.1186/s12879-016-1533-z.

[10] H. Engelberg-Kulka, B. Sat, M. Reches, S. Amitai, R. Hazan, Bacterial programmed cell death systems as targets for antibiotics, Trend. Microbiol. 12 (2004) 66-71.

[11] T. Mukherjee, H. Boshoff, Nitroimidazoles for the treatment of TB: past, present and future, Future Med. Chem. 3 (2011) 1427-1454.

[12] V. Balasubramanian, S. Solapure, H. Iyer, A. Ghosh, S. Sharma, P. Kaur, et al., Bactericidal activity and mechanism of action of AZD5847, a novel oxazolidinone for treatment of tuberculosis, Antimicrob. Agents Chemother. 58 (2014) 495-502.

[13] D.C. Hooper, G.A. Jacoby, Topoisomerase inhibitors: fluoroquinolone mechanisms of action and resistance, Cold Spring Harb. Perspect. Med. 6 (2016), http://dx.doi. org/10.1101/cshperspect.a025320 pii: a025320.

[14] A.E. Grzegorzewicz, J. Kordulakova, V. Jones, S.E. Born, J.M. Belardinelli, A. Vaquie, et al., A common mechanism of inhibition of the Mycobacterium tuberculosis mycolic acid biosynthetic pathway by isoxyl and thiacetazone, J. Biol. Chem. 287 (2012) 38434-38441.

[15] A.R. Baulard, J.C. Betts, J. Engohang-Ndong, S. Quan, R.A. McAdam, P.J. Brennan, et al., Activation of the pro-drug ethionamide is regulated in mycobacteria, J. Biol Chem. 275 (2000) 28326-28331.

[16] D. Jelic, R. Antolovic, From erythromycin to azithromycin and new potential ribosome-binding antimicrobials, Antibiotics 5 (2016) 29, http://dx.doi.org/10. 3390/antibiotics5030029.

[17] S. Chakraborty, T. Gruber, C.E. Barry, H.I. Boshoff, K.Y. Rhee, Para-aminosalicylic acid acts as an alternative substrate of folate metabolism in Mycobacterium tuberculosis, Science 339 (2013) 88-91.

[18] M.C. Cholo, H.C. Steel, P.B. Fourie, W.A. Germishuizen, R. Anderson, Clofazimine: current status and future prospects, J. Antimicrob. Chemother. 67 (2012) 290-298.

[19] A. Alahari, L. Alibaud, X. Trivelli, R. Gupta, G. Lamichhane, R.C. Reynolds, et al., Mycolic acid methyltransferase, MmaA4, is necessary for thiacetazone sus-ceptibility in Mycobacterium tuberculosis, Mol. Microbiol. 71 (2009) 1263-1277.

[20] L. D'Ambrosio, R. Centis, G. Sotgiu, E. Pontali, A. Spanevello, G.B. Migliori, New anti-tuberculosis drugs and regimens: 2015 update, ERJ Open Res. 1 (2015) org/ 10.1183/23120541.00010-2015.

[21] R.S. Wallis, R. Dawson, S.O. Friedrich, A. Venter, D. Paige, T. Zhu, et al., Mycobactericidal activity of Sutezolid (PNU-100480) in sputum (EBA) and blood (WBA) of patients with pulmonary tuberculosis, Plos One 9 (2014) e94462org/ 10.1371/journal.pone.0094462.

[22] R. Nikolay, S. Schmidt, R. Schlomer, E. Deuerling, K.H. Nierhaus, Ribosome assembly as antimicrobial target, Antibiotics 5 (2016) 18, http://dx.doi.org/10.3390/ antibiotics5020018.

[23] M. Wang, Y. Yu, C. Liang, A. Lu, G. Zhang, Recent advances in developing small molecules targeting nucleic acid, Int. J. Mol. Sci. 17 (2016) 779, http://dx.doi.org/ 10.3390/ijms17060779. 
[24] K.M. Evans-Roberts, L.A. Mitchenall, M.K. Wall, J. Leroux, J.S. Mylne, A. Maxwell, DNA, Gyrase is the target for the Quinolone drug Ciprofloxacin in Arabidopsis thaliana, J. Biol. Chem. 291 (2016) 3136-3144.

[25] K. Hards, J.R. Robson, M. Berney, L. Shaw, D. Bald, A. Koul, et al., Bactericidal mode of action of bedaquiline, J. Antimicrob. Chemother. 70 (2015) 2028-2037.

[26] A. Khusro, C. Aarti, TB-PACTS: a fresh emphatic data sharing approach, Asian Pac. J. Trop. Dis. 7 (2017) 97-98.

[27] WHO, Global tuberculosis report 2017, WHO, Geneva, Switzerland, 2017.

[28] M.D. Seo, H.S. Won, J.H. Kim, T. Mishig-Ochir, B.J. Lee, Antimicrobial peptides for therapeutic applications: a review, Molecules 17 (2012) 12276-12286.

[29] R.M. Epand, H.J. Vogel, Diversity of antimicrobial peptides and theirmechanisms of action, BBA-Biomembr 1462 (1999) 11-28.

[30] M. AlMatar, E.A. Makky, G. Yakıcı, I. Var, B. Kayar, F. Köksal, Antimicrobial peptides as an alternative to anti-tuberculosis drugs, Pharmacol. Res. (2017), http://dx. doi.org/10.1016/j.phrs.2017.10.011.

[31] P. Mendez-Samperio, Role of antimicrobial peptides in host defense against mycobacterial infections, Peptides 29 (2008) 1836-1841.

[32] C.M. Linde, S.E. Hoffner, E. Refai, M. Andersson, In vitro activity of PR-39, a prolinearginine-rich peptide, against susceptible and multi-drug-resistant Mycobacterium tuberculosis, J. Antimicrob. Chemother. 47 (2001) 575-580.

[33] J. Carroll, L.A. Draper, P.M. O'Connor, A. Coffey, C. Hill, R.P. Ross, et al., Comparison of the activities of the lantibiotics nisin and lacticin 3147 against clinically significant mycobacteria, Int. J. Antimicrob. Agents 36 (2010) 132-136.

[34] Y.B. Li, Y.Y. Xie, N.N. Du, Y. Lu, H.Z. Xu, B. Wang, Synthesis and in vitro antitubercular evaluation of novel sansanmycin derivatives, Bioorg. Med. Chem. Lett. 21 (2011) 6804-6807.

[35] M. Abedinzadeh, M. Gaeini, S. Sardari, Natural antimicrobial peptides against Mycobacterium tuberculosis, J. Antimicrob. Chemother. 70 (2015) 1285-1289.

[36] L.M. Yin, M.A. Edwards, J. Li, C.M. Yip, C.M. Deber, Roles of hydrophobicity and charge distribution of cationic antimicrobial peptides in peptide-membrane interactions, J. Biol. Chem. 287 (2012) 7738-7745.

[37] Y.B. Huang, J.F. Huang, Y.X. Chen, Alpha-helical cationic antimicrobial peptides: relationships of structure and function, Protein Cell 1 (2010) 143-152.

[38] L. Chen, S.D. Harrison, Cell-penetrating peptides in drug development: enabling intracellular targets, Biochem. Soc. Trans. 35 (2007) 821-825.

[39] R. Montaser, V.J. Paul, H. Luesch, Pitipeptolides C-F, antimycobacterial cyclodepsipeptides from the marine cyanobacterium Lyngbya majuscule from guam, Phytochemistry 72 (2011) 2068-2074.

[40] L. Ivanova, E. Skjerve, G.S. Eriksen, S. Uhlig, Cytotoxicity of enniatins A, A1, B, B1, B2 and B3 from Fusarium avenaceum, Toxicon 47 (2006) 868-876.

[41] S. Sharma, I. Verma, G.K. Khuller, Antibacterial activity of human neutrophil peptide-1 against Mycobacterium tuberculosis H37Rv: in vitro and ex vivo study, Eur. Respir. J. 16 (2000) 112-117.

[42] M.D. Dyer, C. Neff, M. Dufford, C.G. Rivera, D. Shattuck, J. Bassaganya-Riera, et al., The human-bacterial pathogen protein interaction networks of Bacillus anthracis, Francisella tularensis, and Yersinia pestis, PLoS One 5 (2010) e12089, , http://dx.doi. org/10.1371/journal.pone.0012089.

[43] L.R. Matthews, P. Vaglio, J. Reboul, H. Ge, B.P. Davis, J. Garrels, et al., Identification of potential interaction networks using sequence-based searches for conserved protein-protein interactions or "interologs", Genome Res. 11 (2001) 2120-2126.

[44] O. Krishnadev, N. Srinivasan, Prediction of protein-protein interactions between human host and a pathogen and its application to three pathogenic bacteria, Int. J. Biol. Macromol. 48 (2011) 613-619.

[45] M. Pulaganti, B. Banaganapalli, C. Mulakayala, S.K. Chitta, C.M. Anuradha, Molecular modeling and docking studies of O-Succinylbenzoate Synthase of $M$. tuberculosis-A potential target for antituberculosis drug design, Appl. Biochem. Biotechnol. 172 (2014) 1407-1432.

[46] W.W. Krajewski, T.A. Jones, S.L. Mowbray, Structure of Mycobacterium tuberculosis glutamine synthetase in complex with a transition-state mimic provides functional insights, Proc. Natl. Acad. Sci. U. S. A. 102 (2005) 10499-10504.

[47] M.J. McPhillie, R. Trowbridge, K.R. Mariner, A.J. O'Neill, A.P. Johnson, I. Chopra, et al., Structure-based ligand design of novel bacterial RNA polymerase inhibitors, ACS Med. Chem. Lett. 2 (2011) 729-734.

[48] P. Perumal, V.P. Pandey, P. Parasuraman, Docking studies on antimicrobial peptides related to Apidaecinia and human histatin against glutamine synthetase and RNA polymerase in Mycobacterium tuberculosis, Asian J. Pharm. Clin. Res. 7 (2014) 195-201.

[49] T. Huo, W. Liu, Y. Guo, C. Yang, J. Lin, Z. Rao, Prediction of host - pathogen protein interactions between Mycobacterium tuberculosis and Homo sapiens using sequence motifs, BMC Bioinforma. 16 (2015) 100, http://dx.doi.org/10.1186/s12859-0150535-y.

[50] Z. Hu, J.H. Hung, Y. Wang, Y.C. Chang, C.L. Huang, M. Huyck, et al., VisANT 3.5: multi-scale network visualization, analysis and inference based on the gene ontology, Nucleic Acids Res. 37 (2009) 115-121.
[51] D. Croft, G. O'Kelly, G. Wu, R. Haw, M. Gillespie, L. Matthews, et al., Reactome: a database of reactions, pathways and biological processes, Nucleic Acids Res. 39 (2011) 691-697.

[52] M. Michaut, S. Kerrien, L. Montecchi-Palazzi, F. Chauvat, C. Cassier-Chauvat, J.C. Aude, et al., InteroPORC: automated inference of highly conserved protein interaction networks, Bioinformatics 24 (2008) 1625-1631.

[53] S. Kerrien, B. Aranda, L. Breuza, A. Bridge, F. Broackes-Carter, C. Chen, et al., The IntAct molecular interaction database in 2012, Nucleic Acids Res. 40 (2012) $841-846$

[54] I. Xenarios, L. Salwinski, X.J. Duan, P. Higney, S.M. Kim, D. Eisenberg, DIP, the database of interacting proteins: a research tool for studying cellular networks of protein interactions, Nucleic Acids Res. 30 (2002) 303-305.

[55] J. Goll, S.V. Rajagopala, S.C. Shiau, H. Wu, B.T. Lamb, P. Uetz, MPIDB: the microbial protein interaction database, Bioinformatics 24 (2008) 1743-1744.

[56] A. Ceol, A. Chatr Aryamontri, L. Licata, D. Peluso, L. Briganti, L. Perfetto, et al., MINT, the molecular interaction database: 2009 update, Nucleic Acids Res. 38 (2010) 532-539.

[57] T.S. Keshava Prasad, R. Goel, K. Kandasamy, S. Keerthikumar, S. Kumar, S. Mathivanan, et al., Human protein reference database-2009 update, Nucleic Acids Res. 37 (2009) 767-772.

[58] C.B. Bhagat, S.K. Tank, P.R. Dudhagara, N.D. Trivedi, U.N. Trivedi, In silico study of target proteins for Mycobacterium tuberculosis, Am. J. Phytomed. Clin. Ther. 2 (2014) 455-462.

[59] A. Orvedahl, S. MacPherson, R. Sumpter Jr., Z. Talloczy, Z. Zou, B. Levine, Autophagy protects against Sindbis virus infection of the central nervous system, Cell Host Microbe 7 (2010) 115-127.

[60] M. Ponpuak, A.S. Davis, E.A. Roberts, M.A. Delgado, C. Dinkins, Z. Zhao, et al. Delivery of cytosolic components by autophagic adaptor protein p62 endows autophagosomes with unique antimicrobial properties, Immunity 32 (2010) 329-341.

[61] J.M. Winget, T. Mayor, The diversity of ubiquitin recognition: hot spots and varied specificity, Mol. Cell 38 (2010) 627-635.

[62] S. Chongruchiroj, P. Kongsawadworakul, V. Nukoolkarn, M. Jaturanpinyo, W. Nosoong-noen, J. Chingunpitak, et al., Protein-Protein docking and molecular dynamics simulations elucidated binding modes of FUBI-p62 UBA complex, Thai J. Pharm. Sci. 39 (2015) 171-179.

[63] D.I. Banerjee, T.P. Gohil, Interaction of antimicrobial peptide with mycolyl transferase in Mycobacterium tuberculosis, Int. J. Mycobacteriol 5 (2016) 83-88.

[64] S.J. Park, W.S. Son, B.J. Lee, Structural overview of toxin-antitoxin systems in infectious bacteria: a target for developing antimicrobial agents, Biochim. Biophys. Acta Proteins Proteom 1834 (2013) 1155-1167.

[65] V.S. Lioy, O. Rey, D. Balsa, T. Pellicer, J.C. Alonso, A toxin-antitoxin module as a target for antimicrobial development, Plasmid 63 (2010) 31-39.

[66] H.R. Ramage, L.E. Connolly, J.S. Cox, Comprehensive functional analysis of Mycobacterium tuberculosis toxin-antitoxin systems: implications for pathogenesis, stress responses, and evolution, PLoS Genet. 5 (2009), http://dx.doi.org/10.1371/ journal.pgen.1000767 e1000767.

[67] V.L. Arcus, P.B. Rainey, S.J. Turner, The PIN-domain toxin-antitoxin array in mycobacteria, Trends Microbiol. 13 (2005) 360-365.

[68] I.G. Lee, S.J. Lee, S. Chae, K.Y. Lee, J.H. Kim, B.J. Lee, Structural and functional studies of the Mycobacterium tuberculosis VapBC30 toxin-antitoxin system: implications for the design of novel antimicrobial peptides, Nucleic Acids Res. 43 (2015) 7624-7637.

[69] L. Miallau, M. Faller, J. Chiang, M. Arbing, F. Guo, D. Cascio, et al., Structure and proposed activity of a member of the VapBC family of toxin-antitoxin systems VapBC-5 from Mycobacterium tuberculosis, J. Biol. Chem. 284 (2009) 276-283.

\section{List of abbreviations}

AIA: Aspirin-intolerant asthma

AMPs: Antimicrobial peptides

ART: Anti-retroviral therapy

ATP: Adenosine triphosphate

HIV: Human Immunodeficiency Virus

MDR-TB: Multidrug-resistant tuberculosis

Mtb: Mycobacterium tuberculosis

$M t b-O S B S:$ Mtb-O-succinylbenzoate synthase

OSBS: $O$-succinylbenzoate synthase

PATH: Program for Appropriate Technology in Health

RNA: Ribonucleic acid

TA: Toxin-antitoxin

$T B$ : Tuberculosis

WHO: World Health Organization

$X D R-T B$ : Extensively drug-resistant tuberculosis 